\title{
Preliminary Analysis of Yeast Communities Associated with the Spontaneous Fermentation of Musalais, a Traditional Alcoholic Beverage of Southern Xinjiang, China
}

\author{
Z. Lixia ${ }^{1,2, *}$, G. Mingfu ${ }^{1}$, G. Dongqi ${ }^{1,2}$, M. Christensen ${ }^{3}$, H. Xujie ${ }^{1,2}$, L. Hongmei $^{1,2}$, F. Yingge $^{1}$, F. Shu ${ }^{1}$ \\ (1) Xinjiang Production \& Construction Corps Key Laboratory of Protection and Utilization of Biological Resources in Tarim \\ Basin, Tarim University, Ala’er, Xinjiang, 843300, PR China \\ (2) Key Laboratory of Local Agricultural and Animal Production in Xinjiang, Tarim University, Ala'er, Xinjiang, 843300, PR \\ China \\ (3) Retired scientist, 6A Jensen St, Palmerston North, New Zealand
}

Submitted for publication: September 2011

Accepted for publication: January 2012

Key words: Musalais, yeast, community

\begin{abstract}
Musalais is a traditional alcoholic beverage made by the Uighur people in southern Xinjiang, China. The initial fermentation juice is obtained by prolonged boiling of local grape juice and grape residues. In the current study, 242 yeast isolates were obtained from 18 samples (grapes, derived starting products, and progressive stages of fermentation), and 20 phenotypes were distinguished, based on colony characteristics on WL nutrient agar. Fifty representative isolates were selected and found to belong to eight genera (based on rRNA gene sequence analysis). Among the non-Saccharomyces species present on the grapes and related derived substrates, Hanseniaspora spp. was the dominant species. However, nearly all of these species were absent in early fermentation. Saccharomyces cerevisiae was not found until the onset of spontaneous fermentation and quickly became the dominant species. The identified yeast community could be used to further develop indigenous yeast strains to serve the traditional technology of Musalais. The production of Musalais, from a starting substrate that has been boiled for 15 hours to kill all, or nearly all, yeast cells, provides fresh insights into the production of ethanol by the fermentation of grape juice.
\end{abstract}

\section{INTRODUCTION}

Musalais is a traditional alcoholic beverage made with local grapes that has been prepared by the Uygur minority people in the Xinjiang province of China using natural fermentation for more than 2000 years. Musalais is an important element of the tradition and folklore of the Daolang culture and has an important role in the local tourist economy. Local people consuming Musalais have few instances of high blood pressure and hyperlipidemia, which may be related to the wine's reported contribution to the prevention of cardiovascular disease (Stein et al., 1999; Folts, 2002; Gorelik et al., 2008). In fact, Musalais is rich in quercetin, rutin, catechin, anthocyanin and resveratrol. Tests in mice showed that Musalais could enhance the immune system, lower cholesterol, and improve the body's antioxidant ability (an unpublished report from Xinjiang Medical University, 2003).

Musalais is produced by a traditional process. Local Hetianhong grapes are pressed and the juice is collected. The grape residues are added to water to prevent drying and to extract colorants, and are then gently boiled for more than fifteen hours in an iron pan in a clay oven. After heating, the mixture is filtered and mixed with the collected grape juice and heated as before. The boiling temperature of the grape substrates is about $92^{\circ} \mathrm{C}$. The solution is allowed to cool overnight to room temperature to form the Musalais initial fermentation juice, before being transferred into earthenware jugs, sealed, and allowed to ferment for around 45 days. The Musalais production process and how it differs from traditional wine production are shown in Fig. 1.

Musalais is made at oases around the Taklamakan Desert, located in the southern part of Xinjiang province in China. The dry climate and sandy soil are very suitable for grape production. The main production region is A'wati, where there are more than 200 traditional Musalais producers. However, the quality of Musalais can be very unpredictable (Lixia \& Xujie, 2008), possibly because of the unknown

\footnotetext{
*Corresponding author: Lixia Zhu, email: judyzhu1@sina.com [Fax: 86-0997-4681612, Tel. 86-15909978793]

Acknowledgements: The authors would like to thank Prof. Zhang Lili, who provided the initial funding for this study. We are grateful to Jin Chen Yan, chairman of the A'wat Scientific Bureau, and Abudurexiti Gayiti, chairman of the A'wat Musalais Association, for their kind help in collecting and transporting the samples. This research was supported by the National Natural Science Foundation of China (Project No. 31060223) and the Xinjiang Production \& Construction Corps Key Laboratory of Protection and Utilization of Biological Resources in Tarim Basin (Project No. NCET-06-0917).
} 
microorganisms surviving in the traditional spontaneous fermentation.

Many studies have investigated indigenous yeasts associated with wine production (Fleet, 1993; Constantí et al., 1997; Fell et al., 2000; Renouf et al., 2005; Romancino et al., 2008). Although there are studies on the development of the Musalais industry (Lixia et al., 2008), the analysis of physicochemical characteristics (Lixia \& Xujie, 2008; Yinping et al., 2009) and investigations of traditional technology (Lixia et al., 2011), there are no published reports on the yeasts associated with Musalais production.

The present study hoped to gain a better understanding of the indigenous yeast community involved in Musalais fermentation.

\section{MATERIALS AND METHODS}

\section{Source material}

Samples of grapes $(G)$, pressed grape juice $(P G J)$, juice boiled with grape residues (JBR), initial fermenting juice (IFJ), and fermentations during a single Musalais spontaneous fermentation (Fig. 1) of around $1000 \mathrm{~L}$ volume were collected aseptically in sterile polythene bags ( $1 \mathrm{~kg}$ for $\mathrm{G}$ ) and bottles (600 $\mathrm{mL}$ for each liquid sample). The samples were put into insulated containers to maintain the temperature at collection, and were transferred to the laboratory within two hours. The samples covered all stages of the spontaneous fermentation. Specific collection times are shown in Table 1.

The physicochemical parameters, including temperature $\left(28^{\circ} \mathrm{C}\right)$, reducing sugar $(211.6 \mathrm{~g} / \mathrm{L})$, total acid $(7.01 \mathrm{~g} / \mathrm{L})$ and $\mathrm{pH}$ (3.66) of the starting substrate (IFJ), indicated that the initial conditions of the Musalais fermentation were close to those of grape juice.

\section{Isolation and enumeration of yeast colonies}

Bean sprout (BS), potato dextrose agar (PDA) and yeast peptone dextrose agar (YPD) were tested for their suitability for the isolation of a wide range of yeasts. BS medium was prepared from $200 \mathrm{~g}$ of bean sprouts that were boiled for $20 \mathrm{~min}$, after which $20 \mathrm{~g}$ of agar and $20 \mathrm{~g}$ of glucose were added. The solution was made up to $1000 \mathrm{~mL}$ with water and sterilised at $121^{\circ} \mathrm{C}$ for $20 \mathrm{~min}$ at $0.1 \mathrm{Mpa}$. PDA was prepared by boiling $300 \mathrm{~g}$ of unpeeled sliced potatoes in water for 30 minutes, followed by straining of the broth through gauze. The volume was made up to $1000 \mathrm{~mL}$ with water. Twenty grams of dextrose and $20 \mathrm{~g}$ of agar powder were added and the medium was sterilised at $121^{\circ} \mathrm{C}$ for $20 \mathrm{~min}$ at $0.1 \mathrm{Mpa}$. YPD contained $1 \%$ yeast extract, $1 \%$ peptone, $2 \%$ glucose and $2 \%$ agar. WLN agar (Amyl Media, Melbourne, VIC, Australia) was used to sort yeast isolates by their colony characteristics.

In a preliminary experiment, a $5 \mathrm{~g} \mathrm{G}$ (grape) sample was rinsed with $45 \mathrm{ml}$ of sterilised saline solution $(0.9 \% \mathrm{NaCl})$, after which the suspension was diluted in a 10 -fold series using sterile saline solution. One hundred microlitres of the appropriate dilutions were spread onto YPD, PDA and the BS medium, followed by incubation at $25^{\circ} \mathrm{C}$ for three days. Colonies with different characteristics were counted and two to seven colonies were isolated. Subsequently, YPD agar was chosen as the most suitable medium for the isolation of yeasts from the different starting materials, as it gave better growth of colonies than BS medium and PDA. Isolates were purified by streaking on YPD and then stored at $4{ }^{\circ} \mathrm{C}$.

All liquid samples were diluted in a 10 -fold series with sterile saline solution, and $100 \mu \mathrm{l}$ of the appropriate dilutions were spread onto YPD and WLN agar plates. After incubation at $25^{\circ} \mathrm{C}$ for three days on YPD and five days on

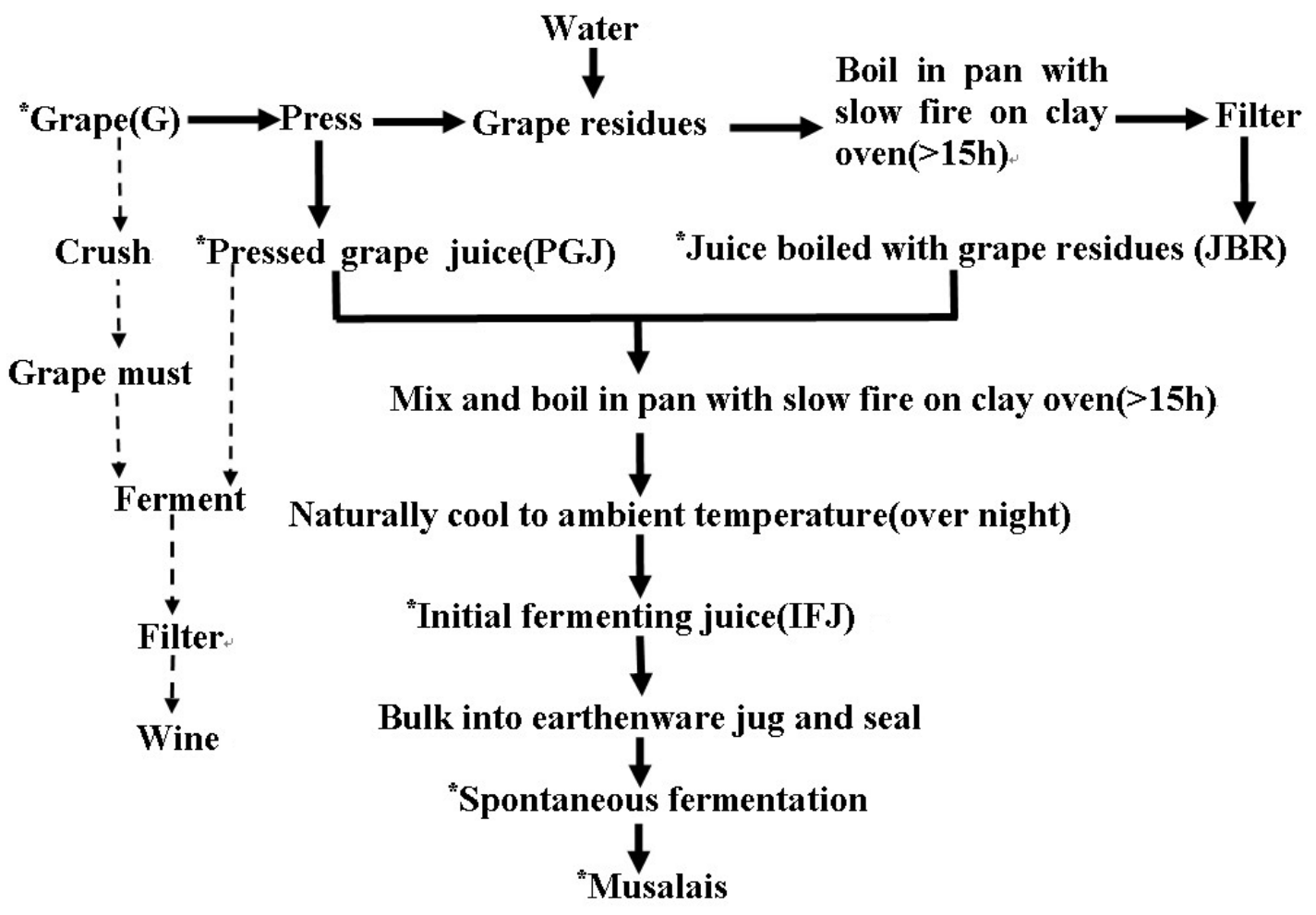

FIGURE 1

The general process of Musalais and wine production. *Samples collected for yeast isolation and enumeration. 


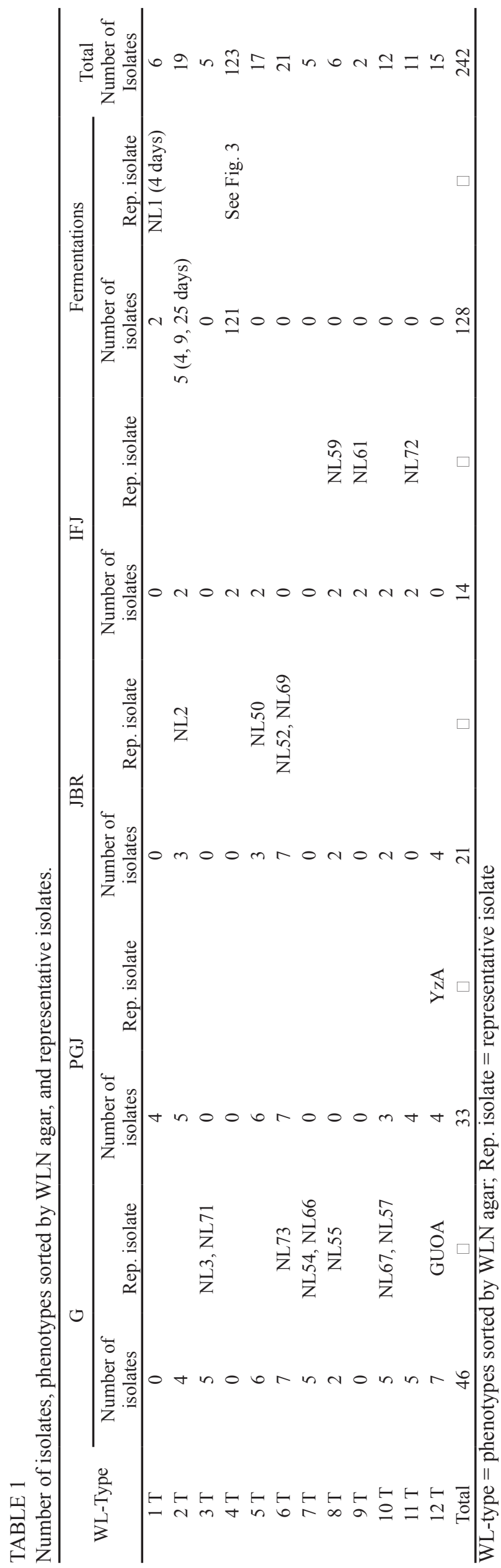

WLN medium, the colonies were counted. Colonies with different characteristics were counted and two to seven colonies were isolated and purified by streaking on YPD isolating medium. The isolates from $\mathrm{G}$ were also purified by the same method. Pure isolates were stored at $4^{\circ} \mathrm{C}$.

Pure isolates were sorted again according to their colony morphology on WLN agar. If two or more phenotypes originally distinguished could no more be separated, they were considered to belong to the same grouping and their total cell counts were combined. Representative isolates from the different phenotypes were selected for $26 \mathrm{~S}$ rRNA gene sequence analysis.

\section{DNA extraction and PCR}

The yeast cells were disrupted by a freeze-boiling method. Yeast cells grown on YPD agar $\left(25^{\circ} \mathrm{C}\right.$ for two to three days $)$ were re-suspended in $100 \mu \mathrm{L}$ of lysis solution $(100 \mathrm{mM}$ Tris-HCL, $30 \mathrm{mM}$ EDTA, $0.5 \%$ SDS), frozen at $-85^{\circ} \mathrm{C}$ for 5 $\mathrm{min}$, and then boiled for $2 \mathrm{~min}$. This procedure was repeated three times. DNA was extracted according to the procedure described by Fengyan et al. (2002).

The amplification was performed in a $50 \mu \mathrm{L}$ mix containing $5 \mu \mathrm{l} 10 \times$ PCR buffer, $3 \mu \mathrm{L} \mathrm{MgCl}_{2} 25 \mathrm{mmol} / \mathrm{L}$, $1 \mu \mathrm{l}$ dNTPs $10 \mathrm{mmol} / \mathrm{L}, 1.5 \mu \mathrm{L}$ DNA polymerase Takara Ex Taq, $1 \mu \mathrm{L} 10$ pmoles DNA template, $1 \mu \mathrm{L} 10$ pmoles each of primers NL1 (5'-GCA TAT CAA TAA GCG GAG GAA AAG-3') and NL4 (5'-GGT CCG TGT TTC AAG ACG G-3') (synthesised by Shanghai Sangon Biological Engineering Technology \& Services Co. Ltd. China.), and $37.5 \mu \mathrm{L}$ double distilled water. Amplification was carried out in an MJ Research PJC-100 thermal cycler (Waltham, MA, USA), as follows: an initial denaturation step of $95^{\circ} \mathrm{C}$ for $5 \mathrm{~min}$; 36 cycles at a denaturation temperature of $94^{\circ} \mathrm{C}$ for $1 \mathrm{~min}$, annealing at $52^{\circ} \mathrm{C}$ for $1 \mathrm{~min}$, and extension at $72^{\circ} \mathrm{C}$ for $20 \mathrm{~s}$ and $1 \mathrm{~min}$; and a final extension at $72^{\circ} \mathrm{C}$ for $8 \mathrm{~min}$. The reactions were maintained at $4{ }^{\circ} \mathrm{C}$ until electrophoretic analysis. The PCR products were sent to the Shanghai Sangon Biological Engineering Technology \& Services Co. Ltd to be purified and sequenced.

\section{Sequence analysis}

The sequences were compared with available sequences in GenBank using the web-based nucleotide-nucleotide BLAST search engine hosted by the National Center for Biotechnology Information, Bethesda, USA (http://www. ncbi.nlm.nih.gov) (Altschul et al., 1997). Typical strains showing identity scores $\geq 99 \%$ were collected, as these levels of similarity are within the values considered to indicate conspecificity (Kurtzman \& Robnett, 1997, 1998).

Sequences of typical strains with high similarity to isolates from this study were aligned using Clustal $\mathrm{X}$ version 1.81 (Thompson et al., 1997). For phylogenetic analysis, regions of sequences with poor alignment were removed. Phylogenetic trees were constructed from distance data transformed according to Kimura (1980), using the neighbour-joining method (Saitou \& Nei, 1987) with a bootstrap of 1000 replicates in MEGA 4. 


\section{RESULTS}

Isolation of yeasts and sorting by characteristics on WLN agar

Based on the colony characteristics of yeasts growing on YPD and WLN agar, 242 isolates were obtained from 18 samples from different stages of Musalais production, including fermentation. They were further sorted into 12 phenotypes by colony characteristics on WLN agar, and 50 representative isolates were selected for identification (Table 1 and Fig. 2).

\section{Identification and phylogeny analysis based on 26S rDNA} The fifty representative yeast isolates were identified by sequencing the D1/D2 domain of the large-subunit (26S) ribosomal DNA. The sequences of the 50 yeast isolates were submitted to GenBank under accession numbers HM191632 to HM191681. The phylogenetic tree was constructed with the 50 isolates and 17 typical strains (Fig. 2).

In the phylogenetic tree (Fig. 2), 32 isolates - NL4, NL5, NL6, NL7, NL8, NL9, NL11, NL12, NL14, NL16, NL17, NL18, NL19, NL20, NL21, NL25, NL26, NL27, NL29, NL30, NL32, NL34, NL38, NL45, NL51, NL60, NL63, NL64, NL74, NL9-9, NL5*, and NL34* - are identified as Saccharomyces cerevisiae, with over 99\% similarity value with the reference strain of $S$. cerevisiae (U44806). Two of these isolates, NL20 and NL34, showed variance from the other isolates, having substitution rates of $1.7 \%$ (nine of 529 nucleotides) and 1.9\% (10 of 529 nucleotides) respectively. This is supported by a comparatively low bootstrap value $(65 \%)$. More variation within the sequences of $26 \mathrm{~S}$ rDNA was revealed among the $32 \mathrm{~S}$. cerevisiae isolates and was revealed over the range of sampling dates (Fig. 3).

The other 18 representative isolates were identified as nonSaccharomyces yeasts, with high matches ( $\geq 96 \%$ bootstrap value), although NL52 and 69, GUOA, NL2, NL61 and NL1 had different branch lengths from their corresponding reference strains. NL50 was identified as Lachancea thermotolerans, NL73 as Hanseniaspora uvarum, NL52 and 69 as $H$. vineae, NL54 and 66 as Meyerozyma guilliermondii (formerly Pichia guilliermondii), NL55 and 59 as Wikerhamomyces anomalus (synonym P. anomala), GUOA as Metschnikowia pulcherrima, YzA as M. fructicola, NL72 as $P$. manshurica, NL67 and 57 as P. membranifaciens, NL2 as Candida zemplinina, NL61 as Issatchenkia hanoiensis, and NL1 as P. kudriavzevii (synonym I. orientalis).

NL71 was identified as P. kluyveri, as it was clustered only with $P$. kluyveri reference strains, as shown in Figs 2 and 4. NL3 clustered with a representative strain of $P$. kluyveri (EF116919) and a reference strain of P. kluyveri var. kluyveri (EF550251) (Fig. 2). It also clustered with 17 P. kluyveri and 10 P. kluyveri var. kluyveri strains (Fig. 4).

Thus, the 50 representative yeasts isolates were ascribed to eight genera and fifteen species.

\section{Yeast community structure during Musalais production, including fermentation}

The 242 isolates obtained were assigned to 12 phenotypes (T) using WLN agar (Table 1). The most common phenotype was $4 \mathrm{~T}$ with 123 isolates, which was obtained following the onset of fermentation. The second most common phenotype was 6T, with 21 isolates. The representative isolates of the two phenotypes were identified as $S$. cerevisiae and Hanseniaspora ssp. respectively (Table 2, Fig. 2, Fig. 3). The distribution and identification of the other 10 yeast phenotypes is also shown in Table 2.

Prior to the onset of fermentation, the yeast species present on the grapes $(G)$ and in the grape juice (PGJ) were all non-Saccharomyces species. The yeasts present in the juice that had been boiled with the residues following the pressing of the grapes (JBR) were also all of non-Saccharomyces species. The first presence of $S$. cerevisiae came from the combined fractions (IFJ) following the overnight cooling after the $15 \mathrm{~h}$ of gentle boiling. In the G, PGJ, JBR and IFJ samples, 12, nine, eight and seven yeast species were detected respectively. The total yeast concentration in the four samples was $1.59 \pm 0.38 \times 10^{5}$ colony-forming units (CFU) per millilitre in G, $3.81 \pm 0.18 \times 10^{7} \mathrm{CFU} / \mathrm{mL}$ in PGJ, $9.25 \pm 0.97 \times 10^{4} \mathrm{CFU} / \mathrm{mL}$ in JBR, and $45.27 \pm 27.86 \mathrm{CFU} /$ $\mathrm{mL}$ in IFJ. During fermentation, the non-Saccharomyces yeasts almost completely disappeared and $S$. cerevisiae became the predominant population.

$S$. cerevisiae was detected in only trace amounts in the initial fermenting juice, but had increased to $3.21 \pm 0.92 \times$ $10^{6} \mathrm{CFU} / \mathrm{mL}$ after four days. The levels detected remained at the $10^{7}$ to $10^{8} \mathrm{CFU} / \mathrm{mL}$ range until day 44 of the fermentation, when the level detected was $3.63 \pm 0.05 \times 10^{6} \mathrm{CFU} / \mathrm{mL}$. The levels decreased over the next 46 days, to $6.00 \pm 0.52 \times 10^{4}$ $\mathrm{CFU} / \mathrm{mL}$ at day 62 and $1.78 \pm 0.52 \times 10^{3} \mathrm{CFU} / \mathrm{mL}$ at day 90 .

Among the non-Saccharomyces species, the $L$. thermotolerans populations ranged from high in the nonboiled substrates to low in the two substrates that had been boiled: $\mathrm{G}\left(1.18 \pm 0.64 \times 10^{4} \mathrm{CFU} / \mathrm{mL}\right)$, PGJ $(3.12 \pm 1.7 \times$ $\left.10^{4} \mathrm{CFU} / \mathrm{mL}\right), \operatorname{JBR}\left(5.02 \pm 0.67 \times 10^{2} \mathrm{CFU} / \mathrm{mL}\right)$, and IFJ $(10.5 \pm 0.7 \mathrm{CFU} / \mathrm{mL})$. C. zemplinina was present in the four starting substrates and was also present on days 4, 9, and 25 . The $M$. pulcherrima and $M$. fructicola population was high on grapes $\left(3.81 \pm 0.49 \times 10^{4} \mathrm{CFU} / \mathrm{mL}\right)$, and survived in PGJ and JBR with populations of $1.8 \pm 0.14 \times 10^{2}$ and $5.00 \pm 1.41$ $\times 10^{2} \mathrm{CFU} / \mathrm{mL}$ respectively. Meyerizyma guilliermondii had a similar concentration $\left(4.0 \pm 1 \times 10^{4} \mathrm{CFU} / \mathrm{mL}\right)$ on grapes as the combined population of Metschnikowia ssp., but was not identified in PGJ and JBR. W. anomalus was present in the G, JBR and IFJ (with just $20 \pm 1.0$ cell counts), but not in PGJ. I. hanoiensis was only present in the initial fermentation sample (cell counts not determined).

$P$. kudriavzevii was isolated from only PGJ and the fermenting sample at day 4 , with $2.01 \pm 0.5 \times 10^{2}$ and $1.12 \pm$ $0.78 \times 10^{4} \mathrm{CFU} / \mathrm{mL}$ respectively. Other Pichia species were distributed in G, PGJ and JBR, but were not detected once fermentation had commenced. All of these Pichia species were associated with grapes, the largest population being that of $P$. manshurica, with $1.06 \pm 0.68 \times 10^{4} \mathrm{CFU} / \mathrm{mL}$. $P$. membranifaciens was present in all four substrates, with a maximum cell number of 1 to $2 \times 10^{2} \mathrm{CFU} / \mathrm{mL}$ being present in $\mathrm{G}$ and PGJ. P. kluyveri and P. kluyveri var. kluyveri were detected only on grapes.

$H$. vineae and H. uvarum were present in G, PGJ and JBR, constituting $99.8 \%$ and $87.8 \%$ percent of the total yeast population in the PGJ and JBR samples respectively. Both species were not detected in the initial fermentation sample. 


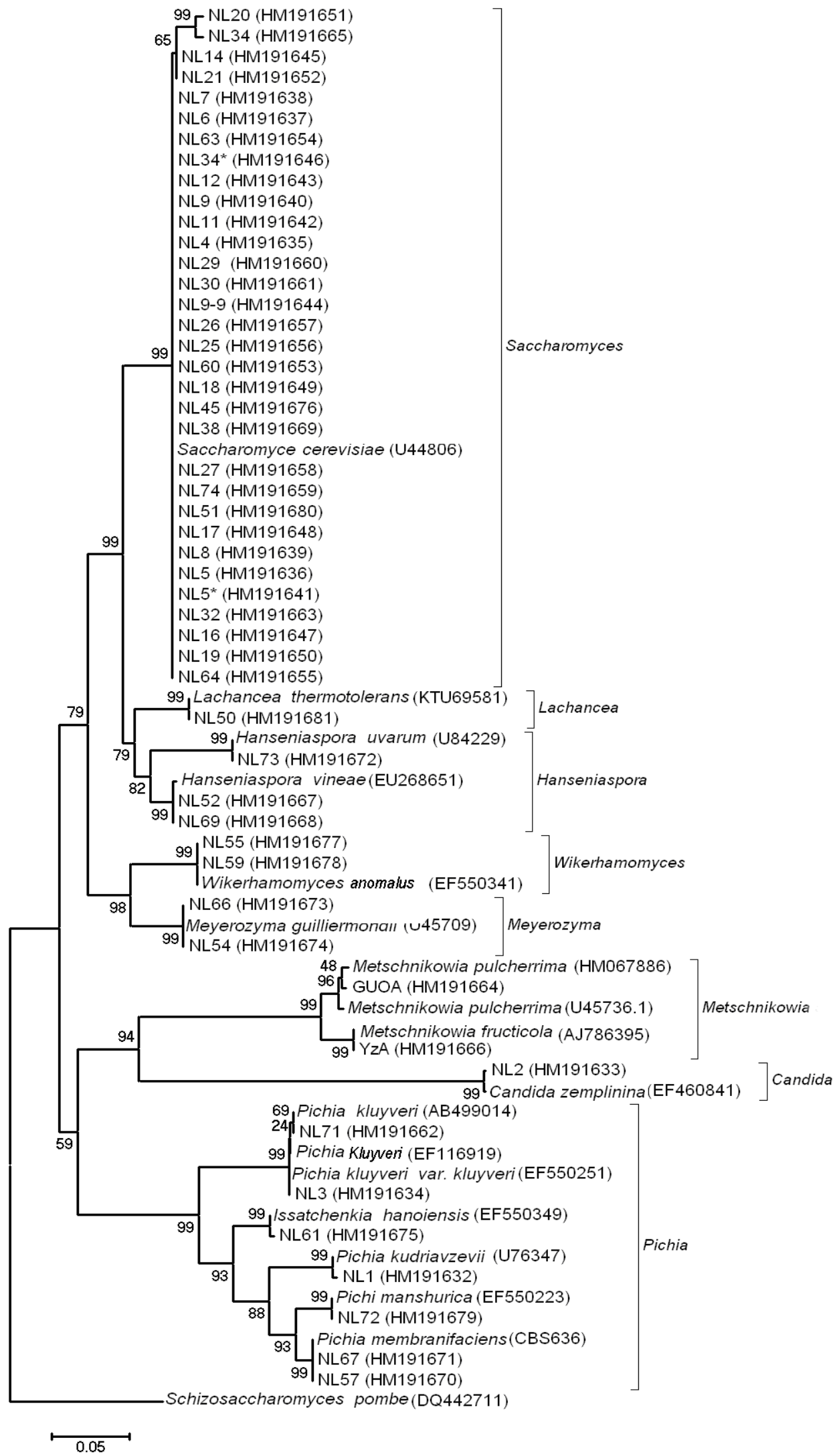

\section{FIGURE 2}

A phylogenetic tree constructed by the neighbour-joining method based on the 26S rRNA D1/D2 domain sequence alignment, showing the relationships of the yeast isolates studied. Bootstrap percentages over $50 \%$ are shown. Schizosaccharomyces pombe was used as the outgroup. The numbers at the nodes indicate the level of bootstrap support based on 1000 replicates. Branch lengths were proportional to the scale given in substitutions per nucleotide. 


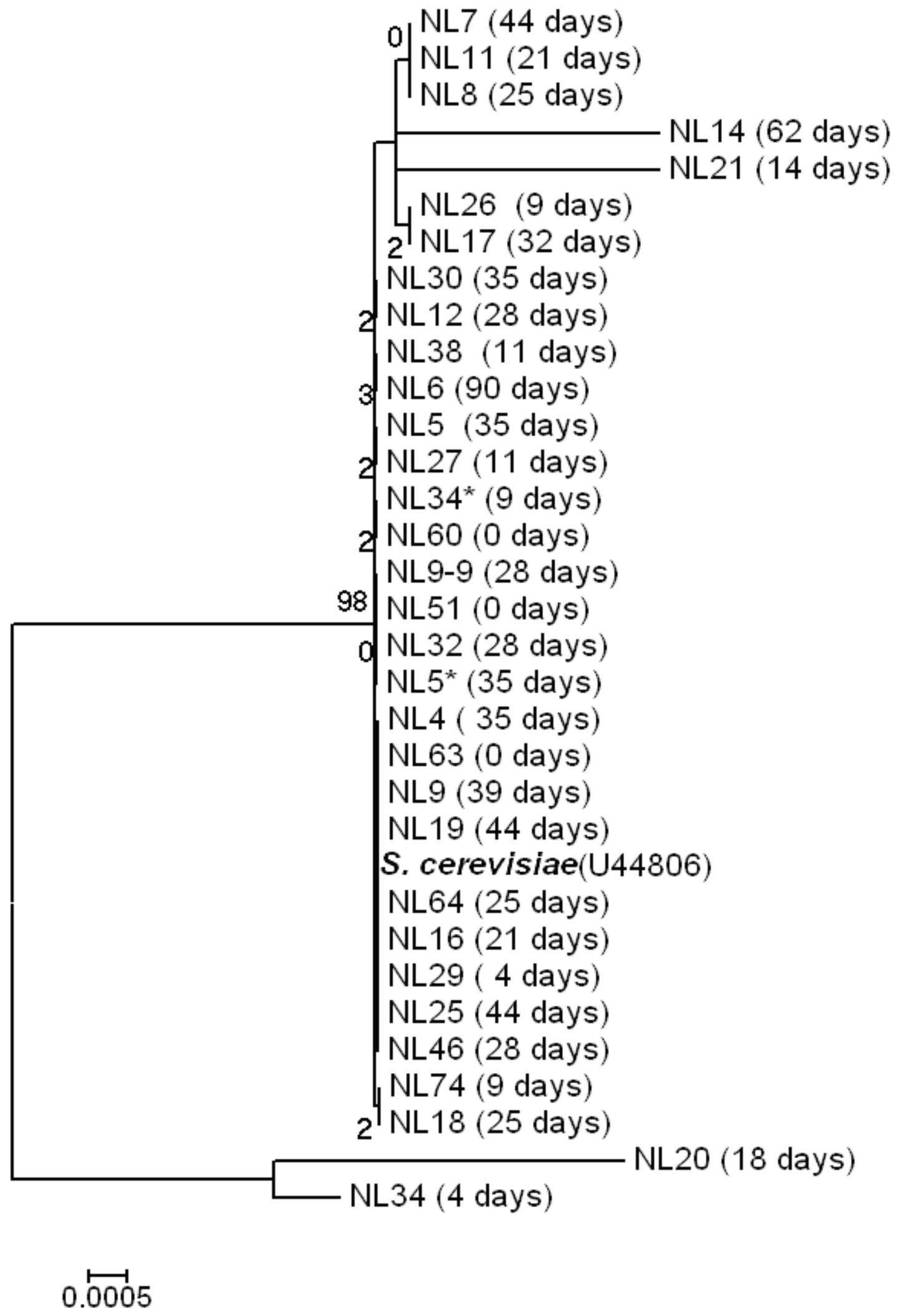

FIGURE 3

The 32 representative isolates of $S$. cerevisiae studied and a typical strain (U44806) were used to construct a phylogenetic tree by the neighbour-joining method based on the 26S rRNA D1/D2 domain sequence alignment, to show the diversity at the strain level. The numbers at the nodes indicate the level of bootstrap support based on 1000 replicates. Branch lengths are proportional to the scale given in substitutions per nucleotide. 


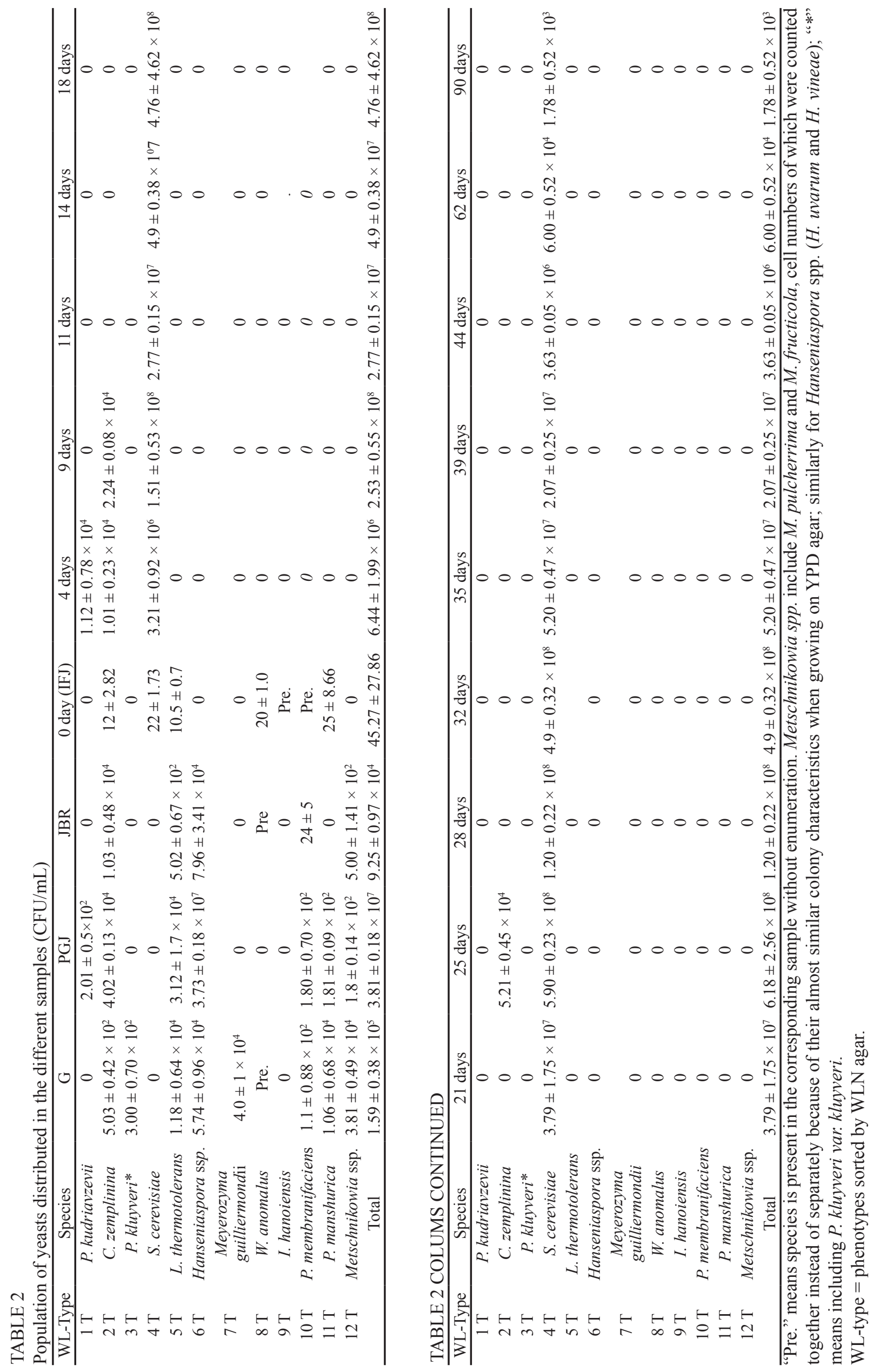




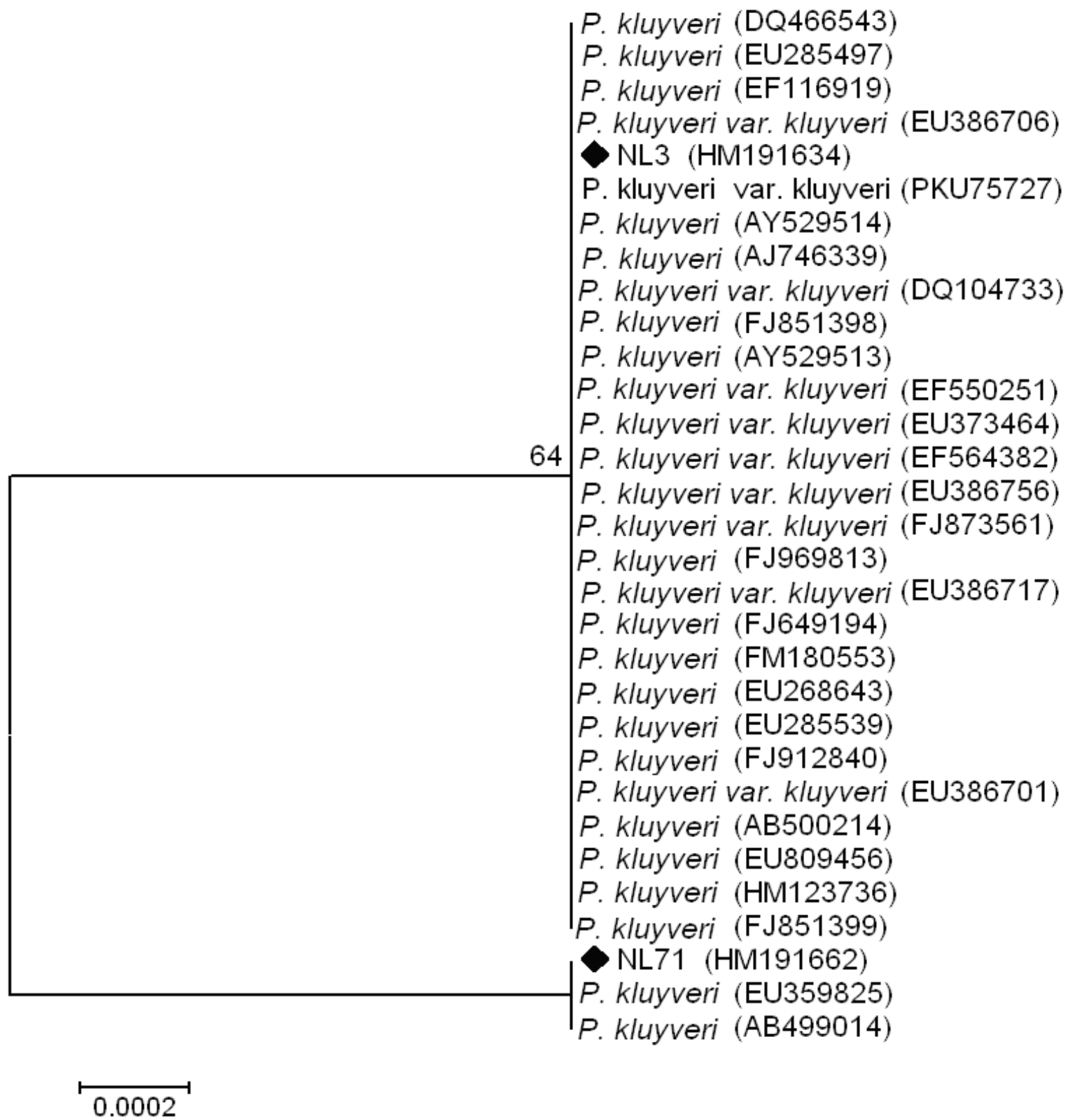

\section{FIGURE 4}

A phylogenetic tree constructed by the neighbour-joining method based on the 26S rRNA D1/D2 domain sequence alignment showing the relations between P. kluyveri and P. kluyveri var. kluyveri (including NL3 and NL71 strains). The numbers at the nodes indicate the level of bootstrap support based on 1000 replicates. Branch lengths are proportional to the scale given in substitutions per nucleotide.

$H$. vineae and $H$. uvarum populations were also high on grapes, being present in the same 10 -fold dilution $\left(10^{4}\right)$ as $L$. thermotolerans, Meyerizyma guilliermondii, Metschnikowia ssp. and P. manshurica.

\section{DISCUSSION}

Yeast communities in the production of Musalais were very diverse at the species and even $S$. cerevisiae strain level, although they were all within the common wine yeast genera (Fleet \& Heard, 1993; Kurtzman, 2003). Not surprisingly, there was a large difference in yeast distribution between the un-boiled and boiled substrates. Almost all of the identified non-Saccharomyces yeasts were present in the pre-fermentation substrates, with Hanseniaspora spp. as the dominant species. However, these non-Saccharomyces yeasts were absent or at low concentrations during fermentation. This was undoubtedly because they did not survive the 15 $\mathrm{h}$ of light boiling in the production of the starting substrate.

There were, however, non-Saccharomyces yeasts present in the two boiled JBR and IFJ substrates. Their presence may be the result of contamination while these two substrates were being slowly cooled. The filtering of the JBR though gauze was a likely entry point for contaminating yeasts. IFJ was not filtered, so airborne and vessel-borne cells were possible means of contamination. Further evidence for the potential of contamination prior to the onset of fermentation comes from the presence of a mycelial mat that developed within the first four days of fermentation. 
S. cerevisiae was only found in the boiled substrates, implying that it must have originated from inside the building where the Musalais was prepared. The means of entry of this yeast species include air, the implements used, and the clay fermentation vessels. Evidence for an airborne inoculum was found by the detection of $S$. cerevisiae in the air of the boiling and fermentation room examined in our study (data not shown). S. cerevisiae entry from the winery instead of the vineyard during wine fermentation has also been reported (Davenport, 1976; Martini \& Martini, 1990; Longo et al., 1991; Constantí et al., 1997). Future studies should include testing for their yeast flora of materials such as earthenware jars and buildings used during the production of Musalais.

$S$. cerevisiae very rapidly became the dominant yeast species during the fermentation of Musalais, possibly because of the boiling of the grape substrates. The boiled starting substrate could provide $S$. cerevisiae with a competitive advantage compared with grape juice or grape must. Boiling might have killed all or most of the yeast and bacteria associated with the grapes, weakening or preventing the competition for nutrients from other microorganisms, and would have increased the concentration of assimilable nitrogen. In addition, boiling would have oxidised phenolic compounds, including tannins, and may have weakened or removed their antimicrobial properties that are associated with wine fermentation (Papadopoulou et al., 2005; Rodríguez Vaquero et al., 2007). In addition, there is no addition of sulphur dioxide $\left(\mathrm{SO}_{2}\right)$ in the processing of Musalais, as is used in traditional wine production to inhibit undesirable microbial growth. This addition of $\mathrm{SO}_{2}$ to wine is also likely to inhibit $S$. cerevisiae (Henick-Kling et al., 1998). It is also possible that local strains of $S$. cerevisiae have a competitive advantage during Musalais production, for example, an ability to tolerate temperatures up to $37^{\circ} \mathrm{C}$ at the vigorous fermentation stage of Musalais production.

The dominance of $S$. cerevisiae, at over $10^{6} \mathrm{CFU} / \mathrm{mL}$, may have prevented the detection of non-Saccharomyces yeasts that might have been present, although at much lower population densities. This was indicated by the failure to persist of the mycelial mat that developed during the first four days of spontaneous fermentation. The concentration of bacilli also remained low (within 100 $\mathrm{CFU} / \mathrm{mL}$ ) during spontaneous fermentation (Lixia et al., 2010). In addition, the key physicochemical parameters are similar to unprocessed grape juice and are indicative of a favourable substrate for $S$. cerevisiae growth. Other factors, for example an increasing concentration of ethanol, high osmotic pressure, and the presence of possible inhibitory substances, also contribute to the strong selective conditions prevailing during the spontaneous fermentation of Musalais. These might explain how the traditional process reliably and successfully completes the spontaneous fermentation of Musalais in practice. This contrasts with wine, where problems with spontaneous fermentation (referred to as stuck or sluggish fermentation) are not uncommon, and where non-Saccharomyces yeasts are the dominant colonisers of the starter grape juice before the dominance of $S$. cerevisiae occurs as the ethanol content increases (Fleet \& Heard, 1993; Linda, 1999; Amato et al., 2006).
C. zemplinina populations fluctuated in a puzzling way during the fermentation. A possible explanation for this is that the inoculum entered the fermentation vessel when it was opened for sampling. Another possible explanation for its absence is the abovementioned strong selective conditions during the fermentation of Musalais.

The results indicate that the bulk of the microbes present in the post-boiling samples occur as a result of contamination from the materials used or from the facility. Further experiments involving multiple fermentations from the same facility or fermentations from other facilities would aid our understanding of this aspect, as would samples taken from the vessels, materials and the fabric of the buildings.

Our results provide an initial indication of the yeast communities associated with the spontaneous fermentation of Musalais. A more comprehensive analysis, involving a wider choice of culture media (e.g. for non-fermentative yeasts), quantitative RT-PCR, restriction fragment length polymorphisms (RFLPs) and denaturing gradient gel electrophoresis (DGGE) directly from the samples instead of cultures, would further identify and quantify the yeast populations present during the various stages of Musalais production. The application of such techniques should also clarify the confusing findings involving $P$. kluyveri and $P$. kluyveri var. kluyveri strains from NCBI, which could not be differentiated based on the 26S rRNA sequences analysis.

\section{CONCLUSIONS}

A yeast community associated with Musalais production has been revealed for the first time. Seven non-Saccharomyces species, including Hanseniaspora spp. as the dominant species, were present on the grapes and related derived substrates; however, nearly all of them were absent in the early fermentation. $S$. cerevisiae was not found until the onset of the spontaneous fermentation stage and quickly became the dominant species. This obviously differs from wine production, during which ethanol-tolerant $S$. cerevisiae gradually dominates fermentation after non-Saccharomyces yeasts initiate fermentation. Diverse $S$. cerevisiae strains were isolated from different fermentation stages. An analysis of the effects of the boiling process involved in the production of Musalais has provided fresh insights into the fermentation of grape juice to produce ethanol.

\section{LITERATURE CITED}

Altschul, S.F., Madden, T.L., Schäffer, A.A., Jinghui, Z., Zheng, Z., Miller, W. \& Lipman, D.J., 1997. Gapped BLAST and PSI-BLAST: a new generation of protein database search programs. Nucl. Acids Res. 25, 33893402 .

Amato, D.D., Corbo, M.R., Nobil, M.A.D. \& Sinigaglia, M., 2006. Effects of temperature, ammonium and glucose concentrations on yeast growth in a model wine system. Int. J. Food Sci. Tech. 41, 1152-1157.

Constantí, M., Poblet, M., Arola, L., Mas, A. \& Guillamón, J.M., 1997. Analysis of yeast populations during alcoholic fermentation in a newly established winery. Am. J. Enol. Vitic. 48, 339-344.

Davenport, R.R., 1976. Distribution of yeasts and yeast-like organisms from aerial surfaces of developing apples and grapes. In: Dickinson, C.H. \& Preecee, T.F. (eds). Microbiology of aerial plant surfaces. Academic Press, London. pp. 325 - 359. 
Fell, J.W., Boekhout, T., Fonseca, A., Gloria, S. \& Statzell-Tallman, A., 2000. Biodiversity and systematics of basidiomycetous yeasts as determined by large-subunit rDNA D1/D2 sequence analysis. Int. J. Syst. Evol. Microbiol. 50, 1351-1371.

Fengyan, B., Jianhua, J. \& Huiyan, L., 2002. Molecular taxonomic study on the problematic Candida strains based on 26s rDNA D1/D2 domain sequence comparison. Mycosystema 1, 27-32.

Fleet, G.H., 1993. The microorganisms of wine making isolation, enumeration and identification. In: Fleet, G.H. (ed). Wine microbiology and biotechnology. Harwood Academic, Singapore. pp. $1-25$.

Fleet, G.H. \& Heard, G., 1993. Yeasts growth during fermentation. In: Fleet, G.H. (ed). Wine microbiology and biotechnology. Harwood Academic, Singapore. pp. $27-54$.

Folts, J.D., 2002. Potential health benefits from the flavonoids in grape products on vascular disease. Adv. Exp. Med. Biol. 505, 95-111.

Gorelik, S., Ligumsky, M., Kohen, R. \& Kanner, J., 2008. A novel function of red wine polyphenols in humans: prevention of absorption of cytotoxic lipid peroxidation products. FASEB J. 22, 41-46.

Henick-Kling, Edinger, Daniel \& Monk, 1998. Selective effects of sulfur dioxide and yeast starter culture addition on indigenous yeast populations and sensory characteristics of wine. J. Appl. Microbiol. 5, 865-876.

Kimura, M., 1980. A simple method for estimating evolutionary rate of base substitutions through comparative studies of nucleotide sequences. J. Mol. Evol. 16, 111-120.

Kurtzman, C.P., 2003. Phylogenetic circumscription of Saccharomyces, Kluyveromyces and other members of the Saccharomycetaceae, and the proposal of the new genera Lachancea, Nakaseomyces, Naumovia, Vanderwaltozyma and Zygotorulaspora. FEMS Yeast Res. 4, 233- 245.

Kurtzman, C.P. \& Robnett, C.J., 1997. Identification of clinically importan ascomycetous yeasts based on nucleotide divergence in the 5 ' end of the large-subunit (26S) ribosomal DNA gene. J. Clin. Microbiol. 35, 12161223 .

Kurtzman, C.P. \& Robnett, C.J., 1998. Identification and phylogeny of ascomycetous yeasts from analysis of nuclear large subunit (26S) ribosomal DNA partial sequence. Antonie van Leeuwenhoek 73, 331-371.

Linda, B.F., 1999. Stuck and sluggish fermentation. Am. J. Enol. Viticult. $50,107-119$.

Lixia, Z. \& Xujie, H., 2008. Investigation on the quality of Musalais grape wine. Liquor-Making Sci. \& Technol. 6, 68-71
Lixia, Z., Miao, H., Dongqi, G., Mingfu, G., Xujie, H.\& Lili, Z., 2010. Isolation, identification and succession of bacteria during spontaneous fermentation of Musalais in Southern Xinjiang. China Brewing 7, 133-136.

Lixia, Z., Shu, F., Dongqi, G., Jincheng, Y. \& Zheng, Y., 2011. The technology of Musalais, a traditional alcohol beverage. In: Hua, L. (ed). Proc. 7th Int. Symp. Viticlut. Enol., April 2011, Xi’an, China. pp. 169 - 171.

Lixia, Z., Xujie, H. \& Qian, X., 2008. Discussion on development strategies of Xinjiang Musalais grape wine. Liquor-Making Sci. \& Technol. 7, 111113

Longo, E., Cansado, J., Agrelo, D. \& Villa, T.G., 1991. Effect of climatic conditions on yeast diversity in grape musts from North West Spain. Am. J. Enol. Vitic. 42, 141-144.

Martini, A. \& Martini, A.V., 1990. Grape must fermentation: past and present. In: Spencer, J.F.T. \& Spencer, D.M. (eds). Yeast technology. Springer-Verlag, Berlin. pp. $105-123$

Papadopoulou, C., Soulti, K. \& Roussis, I.G., 2005. Potential antimicrobial activity of red and white wine phenolic extracts against strains of Staphylococcus aureus, Escherichia coli and Candida albicans. Food Technol. Biotech. 43, 41-46.

Renouf, V., Claisse, O. \& Lonvaud-Funel, A., 2005. Understanding the microbial ecosystem on the grape berry surface through numeration and identification of yeast and bacteria. Aust. J. Grape Wine Res. 11, 316-327.

Rodríguez Vaquero, M.J., Alberto, M.R. \& Manca de Nadra, M.C., 2007. Antibacterial effect of phenolic compounds from different wines. Food Control 2, 93-101.

Romancino, D.P., Di Maio, S., Muriella, R.\& Oliva, D., 2008. Analysis of non-Saccharomyces yeast populations isolated from grape musts from Sicily (Italy). J. Appl. Microbiol. 105, 2248-2254.

Saitou, N. \& Nei, M., 1987. The neighbor-joining method: a new method for reconstructing phylogenetic trees. Mol. Biol. Evol. 4, 406-425.

Stein, J.H., Keevil, J.G., Wiebe, D.A., Aeschlimann, S.\& Folts, J.D., 1999. Purple grape juice improves endothelial function and reduces the susceptibility of LDL cholesterol to oxidation in patients with coronary artery disease. Circulation 100, 1050-1055.

Thompson, J.D., Gibson, T.J., Plewniak, F., Jeanmougin, F. \& Higgins, D.G., 1997. The CLUSTAL X windows interface: flexible strategies for multiple sequence alignment aided by quality analysis tools. Nucl. Acids Res. 24, 4876-4882.

Yinping, L., Cong, L., Weijtm, D., Laaxin, G., Huizhong, Z. \& Ming, G. 2009. Analysis of trace elements in Musalais wine. China Brewing 2, 150 151 